\title{
Symmetry of Ignorance, Social Creativity, and Meta-Design
}

\author{
Gerhard Fischer \\ Center for LifeLong Learning \& Design (L3D) \\ Department of Computer Science and Institute of Cognitive Science \\ University of Colorado, Boulder \\ gerhard@cs.colorado.edu
}

\begin{abstract}
Complex design problems require more knowledge than any one single person can possess, and the knowledge relevant to a problem is often distributed and controversial. Rather than being a limiting factor, "symmetry of ignorance" can provide the foundation for social creativity. Bringing different points of view together and trying to create a shared understanding among all stakeholders can lead to new insights, new ideas, and new artifacts. Social creativity can be supported by new media that allow owners of problems to contribute to the framing and the solving of these problems. These new media need to be designed from a meta-design perspective creating environments in which stakeholders can act as designers and merely be consumers.
\end{abstract}

\section{Keywords}

Conceptual frameworks for creativity and cognition, computers as catalysts for human creativity, fostering creativity in the community, impact of new media on design

\section{INTRODUCTION}

Creativity is often associated with art, but our research work in the Center for LifeLong Learning \& Design (http://www.cs.colorado.edu/ 13d/) is concerned with creativity that is required in everyday work practice by emphasizing the importance of lifelong learning during these activities. The analysis of everyday design practices has shown that knowledge workers and designers have to engage in creative activities to cope with the unforeseen complexities of everyday, real-world tasks. This type of creativity is in most cases not historical; that is, the activity or the product is not necessarily novel or original to a community of practice or society as a whole, but psychological. It is personally novel and meaningful to the stakeholders who produced it [Boden, 1991]. Although analyzing outstanding creative people contributes toward establishing a framework for creativity [Gardner, 1993], understanding creativity in the context of everyday activities is equally important for letting people become more productive and create better work products. This paper explores three interrelated concepts: symmetry of ignorance, social creativity, and meta-design. These concepts are illustrated in the context of the Envisionment and Discovery Collaboratory, an immersive environment supporting these three concepts.

\section{SYMMETRY OF IGNORANCE}

"The clashing point of two subjects, two disciplines, two cultures ought to produce creative chaos." - C.P. Snow

The predominant activity in designing complex systems is that participants teach and instruct each other [Greenbaum \& Kyng, 1991]. Because complex problems require more knowledge than any single person possesses, communication and collaboration among all the involved stakeholders are necessary. For example, domain experts understand the practice, and system designers know the technology. Communication breakdowns are often experienced because stakeholders belonging to different cultures [Snow, 1993] use different norms, symbols, and representations. Rather than viewing this "symmetry of ignorance" [Rittel, 1984] (or "asymmetry of knowledge") as an obstacle during design, we view it as an opportunity for creativity. Having different viewpoints helps one discover alternatives and can help uncover tacit aspects of problems.

When a domain reaches a point where the knowledge for skillful professional practice cannot be acquired in a decade, specialization will increase, collaboration will become a necessity, and practitioners will make increasing use of reference aids, such as printed and computational media supporting external cognition. Design [Simon, 1996] is one such domain par excellence. Complexity in design arises from the need to synthesize different perspectives of a problem, the management of large amounts of information relevant to a design task, and understanding the design decisions that have determined the long-term evolution of a designed artifact. Design problems are wicked and ill-defined; they are moving targets that often do not have solutions but only resolutions [Arias, 1995], and the context in which these problems exist is by nature characterized by change, conflict, and multiple stakeholders. In many cases, the best we can strive for is not consensus, but informed compromises emerging from the conflicting arguments and goals among stakeholders. 
To exploit the symmetry of ignorance requires putting owners of problems in charge [Fischer, 1994b], which will promote direct and meaningful interaction involving people in decisions that affect them. In order to bring important perspectives to the process of design, all stakeholders in the process should be designers and co-developers, not just consumers [Fischer, 1998a]. End-users, as owners of problems, bring special perspectives to collaborative design activities that are of special importance for the framing of problems. The "symmetry of ignorance" requires creating spaces and places that serve as boundary objects where different cultures can meet. Boundary objects serve as externalizations that capture distinct domains of human knowledge and they have the potential to lead to an increase in socially shared cognition and practice [Resnick et al., 1991].

Accepting that most design problems are characterized by a "symmetry of ignorance" leads to a different view of learning. In situations for which the knowledge does not exist, it cannot simply be passed on by the people who have it to the people who need it. Therefore, approaches are required that view learning as collaborative knowledge construction [Scardamalia \& Bereiter, 1994]. This view is in sharp contrast to the teaching cultures of our schools [Illich, 1971], by which teaching is often "fitted into a mold in which a single, presumably omniscient teacher explicitly tells or shows presumably unknowing learners something they presumably know nothing about" [Bruner, 1996]. A critical challenge is to reformulate and reconceptualize this impoverished and misleading conception. Such a teaching culture may be realistic for the early grades in schools [Hirsch, 1996], but it is obviously inadequate for learning processes for which knowledge is distributed among many stakeholders and "the answer" does not exist or is not known. Historically, the roles of teacher and learner were associated with a person, whereas in settings characterized by the "symmetry of ignorance," being a teacher or being a learner is associated only with a specific context. "Official" teachers should feel comfortable becoming learners in many situations.

\section{SOCIAL CREATIVITY}

The power of the unaided, individual mind is highly overrated - "the Renaissance scholar does not exist anymore". Much of our intelligence and creativity results from exploiting the "symmetry of ignorance" as a source of power. Although creative individuals are often thought of as working in isolation, the role of interaction and collaboration with other individuals is critical [Engelbart, 1995]. Creative activity grows out of the relationship between an individual and the world of his or her work, and out of the ties between an individual and other human beings.

\section{A Group Has No Head}

Distributed cognition [Norman, 1993] emphasizes that the heart of intelligent human performance is not the individual human mind but groups of minds in interaction with each other and minds in interactions with tools and artifacts. It is important to understand the fundamental difference of distributed cognition as it operates for the aided individual human mind. Distributed cognition between the individual human mind and artifacts (such as memory systems) often functions well, because the required knowledge an individual needs is distributed between her/his head and the world (for example: an address book, a folder system of email messages, a file system). But a group has no head therefore externalizations are critically more important for social creativity. Externalizations (1) create a record of our mental efforts, one that is "outside us" rather than vaguely in memory; and (2) represent situations that can talk back to us, critique, and negotiate. To make social creativity a reality, we need new forms of knowledge creation, integration, and dissemination. The scarce resource in the information age is not information, but human resources to attend to this information [Simon, 1996].

\section{Knowledge Creation}

One aspect of supporting organizations and groups in creating knowledge is the externalization of an individual's tacit knowledge [Polanyi, 1966]. This is important for three reasons: (1) externalization causes us to begin to move from vague mental conceptualizations of an idea to a more concrete representation of it; (2) externalization provides a means for others to interact with, react to, negotiate around, and build upon it; and (3) externalization provides an opportunity to create a common language of understanding. The use of external representations [Bruner, 1996] serves to focus discussions upon relevant aspects of the framing and understanding of the problem being studied [Arias et al., 1997] and allows stakeholders to engage in a "conversation with the materials" of the design problem [Schön, 1983]. The ability to interact with the problem at hand and to have that situation "talk back" is a crucial mode of design. A principal challenge for social creativity is to capture a significant portion of the knowledge generated by work done within a community. Experiences with organizational memories and collaborative work have exposed two barriers to capturing information: (1) individuals must perceive a direct benefit in contributing to organizational memory that is large enough to outweigh the effort [Grudin, 1989]; and (2) the effort required to contribute to organizational memory must be minimal so it will not interfere with getting the real work done [Carroll \& Rosson, 1987].

\section{Knowledge Integration}

The challenge is to integrate the various perspectives emerging from the "symmetry of ignorance" among articulate stakeholders. By supporting the process of reflection within a shared context defined by the task at hand, opportunities can emerge from enhancing the creation of shared understanding. This process melds the information that is collaboratively constructed into the problem-solving context, informing the process as well as the stakeholders and allowing them to participate from a more enriched and meaningful perspective [Brown et al., 


\begin{tabular}{|l|l|}
\hline Concept & Implications \\
\hline convivial tools & $\begin{array}{l}\text { allow users to invest the world with their meaning and to use tools for the } \\
\text { accomplishment of a purpose they have chosen [Illich, 1973] }\end{array}$ \\
\hline human problem-domain interaction & bring task to the forefront; provide time on task [Fischer, 1994a] \\
\hline critiquing & increase the back-talk of the artifacts [Fischer \& Nakakoji, 1992] \\
\hline open, evolvable systems & put owners of problems in charge [Fischer, 1994b] \\
\hline underdesigned systems & create seeds and constructs for design support at use time [Fischer, 1998b] \\
\hline
\end{tabular}

Figure 1: Concepts of Meta-Design

1994]. It also enhances the quality of the designed artifact due to the synergy of interaction that draws out ideas and perspectives in a conversational manner. The resulting, richly contextualized information is available for future stakeholders to draw upon, informing them, not only about the surface level of the design, but about the deeper characteristics behind the design. Collaborative constructions result in work products that are enriched by the multiple perspectives of the participants. The information repositories and organizational memories that are created in these ways are no longer very large, impenetrable "write-only" stores, but are actively integrated into the work processes and social practices of the community that constructs them.

\section{Knowledge Dissemination}

Humans seldom (if at all) explore large reflection spaces (e.g., thousands of pages of documentation, design rationales, argumentation, etc.) in the abstract [Moran \& Carroll, 1996], but do so to obtain information in response to breakdowns [Fischer, 1994c] occurring in their design activities. Making information relevant to the task at hand (rather than drowning users in decontextualized information) and supporting the interaction of multiple perspectives and the various strengths that each stakeholder brings to the task allows collaborative exploration of the knowledge and shared understanding of the problem. The knowledge is made to serve the process of collaborative design by providing "the "right' information at the 'right' time and in the 'right' way."

\section{META-DESIGN}

A Chinese proverb says: "If you give a fish to a human, you will feed him for a day - if you give someone a fishing rod, you will feed him for life." This saying can be extended by arguing that "if we can provide someone with the knowledge, the skill, and the tools for making a fishing rod, we can feed the whole community." Meta-design
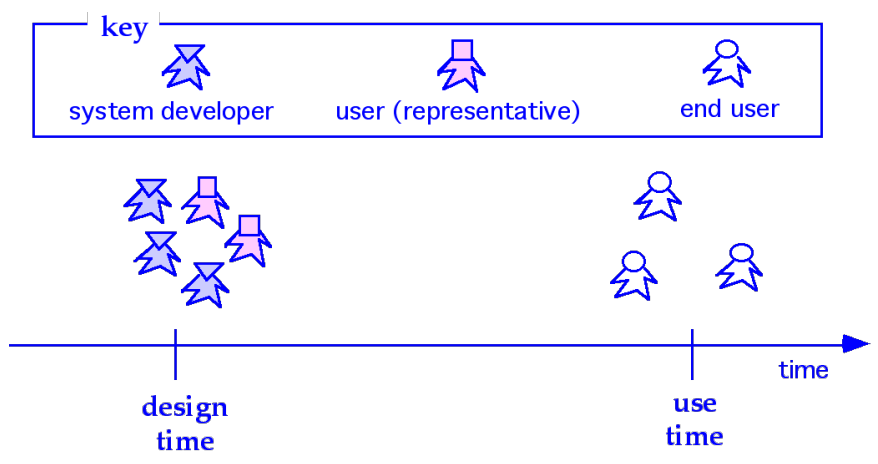

characterizes activities, processes, and objectives to create new media and environments that allow users to act as designers and be creative. This can be compared with the objective in art that focuses on the artist as the facilitator of the creative experience for users. In our work, we have explored a set of concepts and ideas for meta-design that are summarized in Figure 1.

We will choose the concept of open, evolvable systems to illustrate some of the aspects and challenges associated with meta-design. A fundamental question we have discussed in our research with collaborating communities (such as urban and transportation planners) has been: "Why do the urban and transportation planners not take advantage of programs such as SimCity?" The answer is because SimCity is a closed system. For example, when users of SimCity encounter a problem with crime in their simulation, there is only one way to resolve the problem build more police stations. Other solutions such as increasing social services were not conceived in the model at design time, and because the system is closed, there is no way to explore these kinds of changes. Although SimCity is a useful educational and recreational object, its closed nature is a significant impediment to using this kind of model for actual city planning. An open system provides opportunities for significant changes to the system at all levels of complexity, allowing emergent resolution of situations in the context of collaborative design. Enhancement and evolution of the system by the owners of the problems must be a "first-class design activity."

The need for meta-design is founded on these observations: design problems in the real-world require open systems that users can modify and evolve. Because problems cannot be completely anticipated at design time (when the system is developed), users at use time will discover mismatches between their problems and the support that a system provides (see Figure 2).

\section{Example: The Envisionment and Discovery Collaboratory}

The Envisionment and Discovery Collaboratory (EDC) [Arias et al., 1997] is a meta-design effort that acknowledge the "symmetry of ignorance" as a fundamental design constraint. It supports social creativity by empowering stakeholders to act as designers, allowing them to create shared understanding, to contextualize information to the task at hand, and to create objects-to-

Figure 2: Design and Use Time 
think-with in collaborative design activities. The EDC framework (http://www.cs.colorado.edu/ 13d/systems/$\mathrm{EDC} /$ ) is applicable to different domains, but our initial effort has focused on the domains of urban planning and decision making, specifically in transportation planning and community development.

The EDC is a meta-design effort to create environments that are immersive and emergent. An immersive environment allows stakeholders to become deeply engaged in problem solving in the context of information, action, reflection, and collaborations relevant to the situation, whereas the term emergent addresses the need for this context to grow and evolve based on ongoing problemsolving activities. These intertwined concepts require the EDC to support stakeholders in (1) creating and capturing knowledge in the context of collaborative design activities; (2) sustaining the timeliness and utility of evolving information; (3) articulating their own knowledge in a form that other people can understand; (4) enhancing existing knowledge with new knowledge; and (5) creating tools that help stakeholders think, and help analyze their constructions and artifacts [Fischer \& Nakakoji, 1992].

Figure 3 shows the current realization of the EDC environment supporting "around-the-table" interaction and contextualizing information in design activities. Individuals using the EDC convene around a computationally enhanced table, shown in the center of the figure. This table serves as the action space for the EDC. Currently realized as a touchsensitive surface, the action space allows users to manipulate the computational simulation projected on the surface by interacting with the physical objects placed on the table. The table is flanked by a second computer that drives the vertical touch-sensitive computational whiteboard serving as the EDC's reflection space. In the figure, users are filling out a Web-based transportation survey in the reflection space that provides in part the information base that is associated with the model being constructed in the action space. The reflection and action spaces are connected by communication between the two computers using the Web as a medium. The entire physical space, through the immersion of people within the representations of the problem-solving task, creates a prototype of an integrated, socio-technical human-computer system. The open nature of the EDC supports integration of new constructions and differing perspectives into the environment. The dissemination of constructed knowledge is afforded through the EDC's WWW linkages between the action and reflection spaces. These constructions can be shared in a distributed manner just as distributed information can be integrated through the reflection space.

The EDC represents a theory-based architecture and process model with three layers: (1) a domain-independent framework and architecture for integrated physical and computational environments that support creating shared understanding through collaborative design; (2) application domains, in which the domain-independent architecture is realized for a specific class of problems (for example, the application domain discussed in [Arias et al., 1997] addresses decision problems of urban planning, specifically for transportation systems); and (3) specific applications created to contextualize an application domain to a concrete situation, such as transportation planning in the city of Boulder.

The "symmetry of ignorance" among the stakeholders in the EDC serves as a source for social creativity by providing users with many opportunities to construct their own situations and have control in the description of a problem. For example: neighbors can change the model and see how their changes affect the transportation system. They can place new buses along a bus route to increase bus frequency along the route; or they can move bus stops, change the bus route by moving appropriate pieces, and modify the behavior of the buses or traffic lights. In the course of framing and solving their problems, neighbors may find that the existing environment does not model some situations in which they are interested. The metadesign allows stakeholders to extend the system to meet the needs of unforeseen situations [Fischer, 1998b].

\section{Computational Substrates Embedded in the EDC}

To exploit the symmetry of ignorance and to support social creativity, the EDC incorporates a large variety of computational mechanisms and substrates. The action space of the EDC is built using AgentSheets [Repenning, 1999], a software environment for creating simulations and

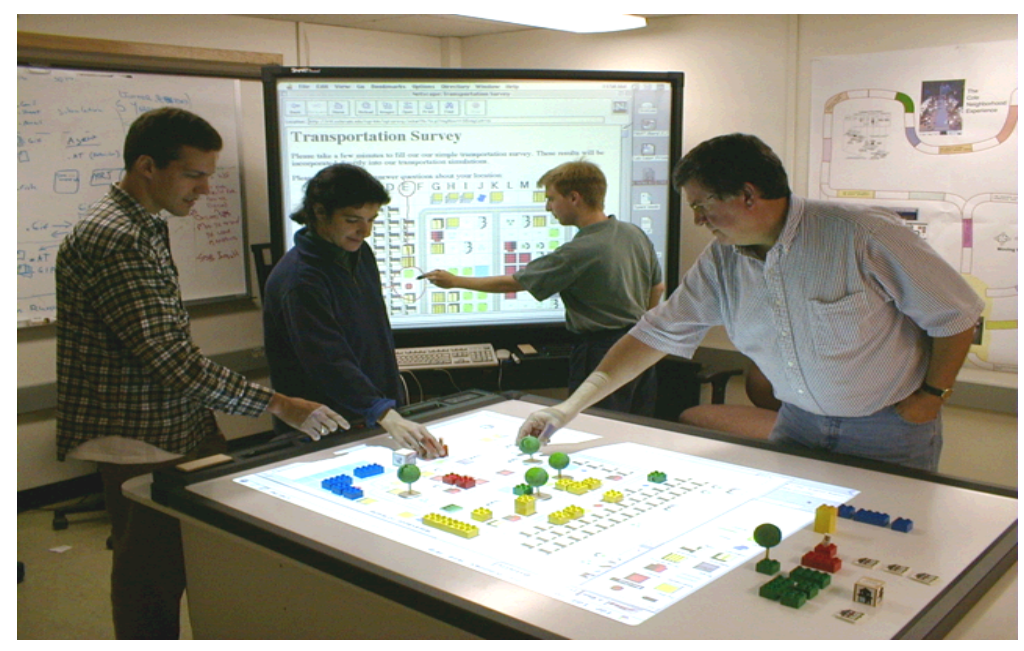

Figure 3: The Current Prototype of the EDC 
domain-oriented environments. AgentSheets applications include a collection of autonomous computational processes, called agents, that are comprised of a look (their on-screen representation), and a programmed behavior. Agents in AgentSheets are programmed in Visual AgenTalk, a programming environment suitable for end- problem.

An important technical challenge for social creativity is to capture the informal, situated problem-solving episodes that real people generate in solving real problems, which formal processes have difficulties to anticipate or to capture. An important non-technical challenge for social

\begin{tabular}{|c|c|c|}
\hline Issue & SimCity & EDC \\
\hline $\begin{array}{l}\text { user-directedness, openness of } \\
\text { systems }\end{array}$ & $\begin{array}{l}\text { rich construction mechanisms; } \\
\text { simulation is a "black" box }\end{array}$ & $\begin{array}{l}\text { rich construction and } \\
\text { modification mechanisms }\end{array}$ \\
\hline contextualized information & no support for task-based indexing & linking of action and reflection \\
\hline $\begin{array}{l}\text { breakdowns as opportunities for } \\
\text { learning }\end{array}$ & $\begin{array}{l}\text { there are no explanations of problem } \\
\text { mechanisms }\end{array}$ & $\begin{array}{l}\text { critiquing leads to relevant argumentation } \\
\text { in the reflection space }\end{array}$ \\
\hline $\begin{array}{l}\text { adapting to expertise and } \\
\text { background knowledge }\end{array}$ & $\begin{array}{l}\text { adjust parameters (amount of money, } \\
\text { simulation speed, turn off disasters) }\end{array}$ & $\begin{array}{l}\text { users adapt system to their perspectives, } \\
\text { goals, needs, and background knowledge }\end{array}$ \\
\hline engagement / motivation & $\begin{array}{l}\text { games are engaging but do not allow } \\
\text { users to model their own situations }\end{array}$ & $\begin{array}{l}\text { owners of problems are in charge, and } \\
\text { engage in self-directed experience }\end{array}$ \\
\hline collaboration & $\begin{array}{l}\text { multi-user version restricted to } \\
\text { mayoral decisions and voting }\end{array}$ & $\begin{array}{l}\text { ability to share argumentation and } \\
\text { simulation components }\end{array}$ \\
\hline
\end{tabular}

Figure 4: Closed versus Open Systems - SimCity versus EDC

user programmers.

The reflection space in EDC is supported by DynaSites [Ostwald, 1999], which allows users to create extensible, Web-based information spaces. DynaSites provides computational support for collaborative working, learning, and knowledge construction by supporting these activities in a way that they can grow and be shaped over time by the people who use them. DynaSites information spaces are dynamic because their pages are built at use time (from a database) whereas typical Web sites are static - their links and displays are determined at design time. They differ from most Web sites because they are dynamic and evolvable by users.

\section{ASSESSMENT}

The EDC environment and its use with specific communities has been an explicit attempt to instantiate and evaluate the adequacy and usefulness of a framework grounded in the concepts of symmetry of ignorance, social creativity, and meta-design. We live in a world where problems often require the collaboration of stakeholders from different communities, each seeing the world from their own perspective, each having their own background knowledge and their cognitive, computational and physical tools and artifacts. Exploiting the symmetry of ignorance as a source of power requires not only a willingness to talk to collaborators, but also externalizations that allow people to think and to argue about and that help them to create incrementally a shared understanding of the design creativity is to take motivation seriously. There must be an incentive to create social capital by rewarding stakeholders to be good citizens by contributing and receiving knowledge as a member of a community.

Meta-design is supported by the seeding, evolutionary growth, reseeding model [Fischer, 1998b], which allows and encourages designers to explicitly underdesign and underprescribe at design time and provide constructs and environments (e.g., Visual AgenTalk and DynaSites, mentioned above) for design support and situated interpretations and actions at use time (see Figure 2). In a closed system, it is difficult or impossible for users to change the system to deal with new and unforeseen situations. System developers control additions and modifications, and when they are no longer present, the system cannot handle a new situation. This implies that users have to work around or outside such a closed system (or perhaps abandon the system altogether) when they encounter an unexpected situation. Figure 4 compares SimCity with the Envisionment and Discovery Collaboratory (EDC) from a meta-design perspective.

\section{Consumers and Designers}

Meta-design is design for designers, not for consumers. By arguing for the desirability for humans to be designers [Fischer, 1998a], it should be stated explicitly that there is nothing wrong with being a consumer and that we can learn and enjoy many things in a consumer role (e.g., listening to a lecture, watching a tennis match, or attending a concert).

Consumer $<$

$>$ Designer

passive consumer

active consumer

end-user

user

power users

domain designer

meta-designer 
It is also a mistake to assume that being a consumer or being a designer would be a binary choice; it is rather a continuum ranging from passive consumer, to active consumer, to end-user, to user, to power users [Nardi, 1993], to domain designer, to medium designer, all the way to meta-designer (see Figure 5). Problems occur (1) when someone wants to be a designer but is forced to be a consumer, and (2) when being a consumer becomes a universal habit and mindset dominating a human life completely.

Our empirical observations and studies have clearly of the macro in the unwrapped form). Although end-user programming and modification components are necessary for meta-design environments, they by themselves are far from sufficient. Our empirical investigations have shown that few users take advantage of the end-user modifiability components provided by environments such as Microsoft Word, and even fewer users engage in exchanging their extensions among each others. Other communities (such as the open source code community [Raymond, 1998] and web-based community of practice [Expert-Exchange, 1999]) are better success examples to be analyzed for meta-

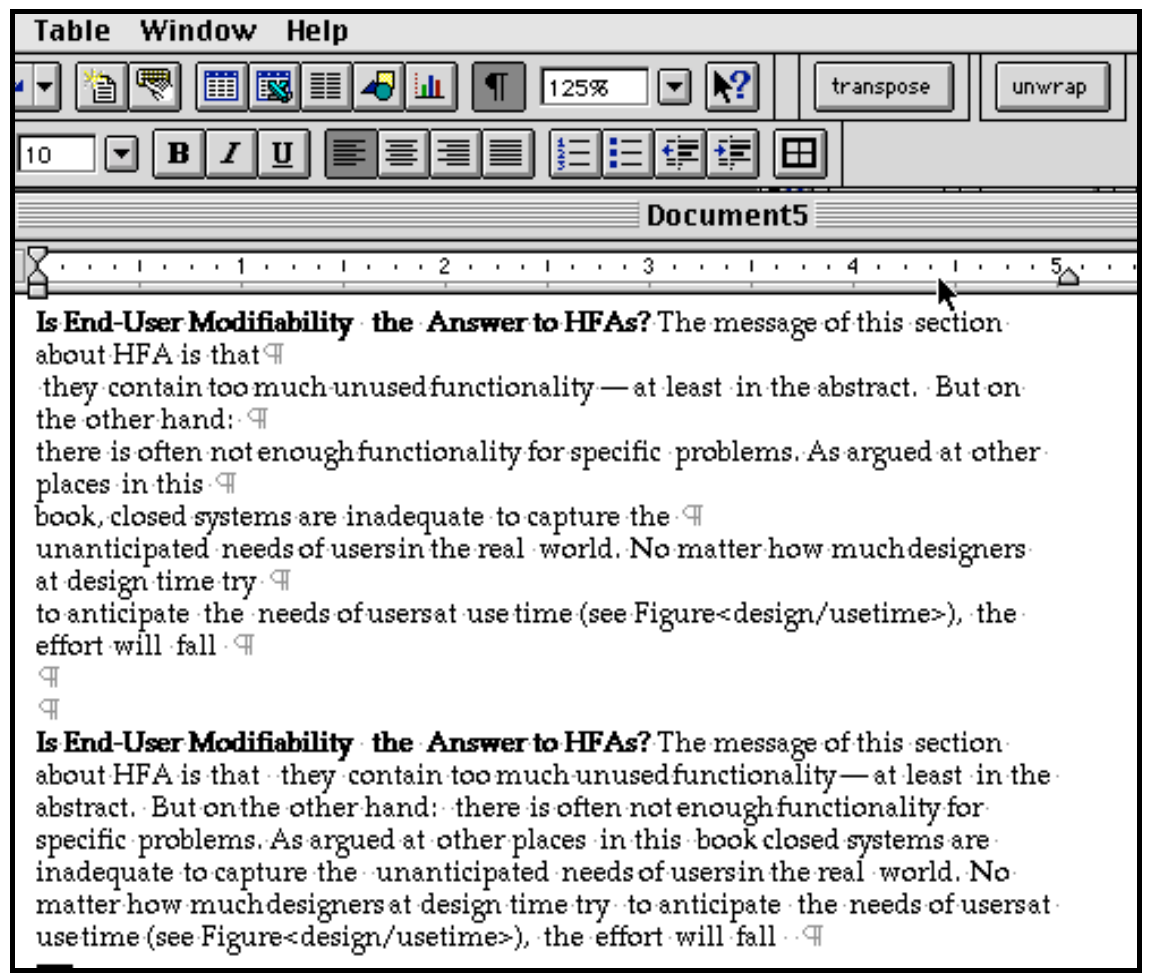

Figure 6: Use of Meta-Design Components in Microsoft Word

demonstrated that meta-design requires more than just technical facilities. The possibility of extending open systems will not take place within the first few days or weeks of using them, but will require the long-term use of a system by owners of problems engaged in the cultivation of a rich repertoire of personally and socially meaningful artifacts. For example, we do not expect all users to become end-user programmers or to be interested in making radical changes to the system. Their contributions will depend on the perceived benefit of contributing, which involves the effort needed to make changes and the utility received for effecting changes.

Figure 6 shows the exploitation of the meta-design support built into Microsoft Word by showing two macros: "transpose," which transposes two characters, and "unwrap," which unwrap texts as shown in the screen image (the same text appears in two forms: in the top half as wrapped text and in the bottom half after the application design and social creativity.

\section{CONCLUSIONS}

Failing to make computation accessible with reasonable cognitive costs to all people will reduce people's creativity. Furthermore it will prevent computational environments that need to evolve through the active contributions from their users [Raymond, 1998] from coming into existence. The three concepts discussed in this paper (symmetry of ignorance, social creativity, and meta-design) provide a conceptual framework for understanding creativity and cognition and for fostering creativity in communities. The EDC was used as an illustration of the impact of this framework. It fosters social creativity by exploiting the symmetry of ignorance to enhance conversations around shared, mutually understandable artifacts, and it allows stakeholders to learn with and from each other. By being a meta-design environment, the EDC allows stakeholders to act as designers, making it possible to deal with new requirements as they emerge during development, and 
thereby contributing to the integration of problem framing and problem solving as an important source for creativity.

\section{ACKNOWLEDGMENTS}

The author would like to thank all the members of the L3D Center for their numerous contributions to this research. Special thanks to Ernie Arias, Hal Eden, Eric Scharff, and Andy Gorman, who have been the major developers of the EDC, and to Andri Ioannidou for helpful comments on an earlier version of this paper. The research was supported by (1) the National Science Foundation, Grants REC-9631396 and IRI-9711951; (2) Software Research Associates, Tokyo, Japan; and (3) PFU, Tokyo, Japan.

\section{REFERENCES}

1. Arias, E. G. (1995) "Designing in a Design Community: Insights and Challenges." In G. M. Olson \& S. Schuon (Eds.), Proceedings of DIS'95 Symposium on Designing Interactive Systems: Processes, Practices, Methods, \& Techniques, ACM Press, New York, pp. 259-263.

2. Arias, E. G., Eden, H., \& Fischer, G. (1997) "Enhancing Communication, Facilitating Shared Understanding, and Creating Better Artifacts by Integrating Physical and Computational Media for Design." In Proceedings of DIS'97: Designing Interactive Systems: Processes, Practices, Methods, \& Techniques, ACM, New York, pp. 1-12.

3. Boden, M. (1991) The Creative Mind: Myths \& Mechanisms, Basic Books.

4. Brown, J. S., Duguid, P., \& Haviland, S. (1994) "Toward Informed Participation: Six Scenarios in Search of Democracy in the Information Age," The Aspen Institute Quarterly, 6(4), pp. 49-73.

5. Bruner, J. (1996) The Culture of Education, Harvard University Press, Cambridge, MA.

6. Carroll, J. M. \& Rosson, M. B. (1987) "Paradox of the Active User." In J. M. Carroll (Ed.) Interfacing Thought: Cognitive Aspects of Human-Computer Interaction, The MIT Press, Cambridge, MA, pp. 80111.

7. Engelbart, D. C. (1995, August 1995) "Toward Augmenting the Human Intellect and Boosting Our Collective IQ," Communications of the ACM, pp. 3033. Expert-Exchange (1999), at http://www.expertsexchange.com.

8. Fischer, G. (1994a) "Domain-Oriented Design Environments," Automated Software Engineering, 1(2), pp. 177-203.

9. Fischer, G. (1994b) "Putting the Owners of Problems in Charge with Domain-Oriented Design Environments." In D. Gilmore, R. Winder, \& F. Detienne (Eds.), User-Centered Requirements for Software Engineering Environments, Springer Verlag, Heidelberg, pp. 297-306.
10. Fischer, G. (1994c) "Turning Breakdowns into Opportunities for Creativity," Knowledge-Based Systems, Special Issue on Creativity and Cognition, 7(4), pp. 221-232.

11. Fischer, G. (1998a) "Beyond 'Couch Potatoes': from Consumers to Designers," Asia Pacific Computer Human Interaction Conference, pp. 2-9.

12. Fischer, G. (1998b) "Seeding, Evolutionary Growth and Reseeding: Constructing, Capturing and Evolving Knowledge in Domain-Oriented Design Environments," Automated Software Engineering, 5(4), pp. 447-464.

13. Fischer, G. \& Nakakoji, K. (1992) "Beyond the Macho Approach of Artificial Intelligence: Empower Human Designers - Do Not Replace Them," Knowledge-Based Systems Journal, Special Issue on AI in Design, 5(1), pp. 15-30.

14. Gardner, H. (1993) Creating Minds, Basic Books, Inc, New York.

15. Greenbaum, J. \& Kyng, M. (Eds.) (1991) Design at Work: Cooperative Design of Computer Systems, Lawrence Erlbaum Associates, Inc., Hillsdale, NJ.

16. Grudin, J. (1989) "Why groupware applications fail: Problems in design and evaluation," Office: Technology and People, 4(3), pp. 245-264.

17. Hirsch, E. D., Jr. (1996) The Schools We Need And Why We Don't Have Them, (First ed.), Doubleday, New York.

18. Illich, I. (1971) Deschooling Society, Harper and Row, New York.

19. Illich, I. (1973) Tools for Conviviality, Harper and Row, New York.

20. Moran, T. P. \& Carroll, J. M. (Eds.) (1996) Design Rationale: Concepts, Techniques, and Use, Lawrence Erlbaum Associates, Inc., Hillsdale, NJ.

21. Nardi, B. A. (1993) A Small Matter of Programming, The MIT Press, Cambridge, MA.

22. Norman, D. A. (1993) Things That Make Us Smart, Addison-Wesley Publishing Company, Reading, MA.

23. Ostwald, J. (1999) DynaSites, at http://www.cs.colorado.edu/ ostwald.

24. Polanyi, M. (1966) The Tacit Dimension, Doubleday, Garden City, NY.

25. Raymond, E. S. (1998) The Cathedral and the Bazaar, at http://earthspace.net/ esr/writings/cathedralbazaar/cathedral-bazaar.html.

26. Repenning, A. (1999) Agentsheets, at http://www .cs.colorado.edu/ 13d/systems/agentsheets/.

27. Resnick, L. B., Levine, J. M., \& Teasley, S. D. (Eds.) (1991) Perspectives on Socially Shared Cognition, 
American Psychological Association, Washington, D.C.

28. Rittel, H. (1984) "Second-Generation Design Methods." In N. Cross (Ed.) Developments in Design Methodology, John Wiley \& Sons, New York, pp. 317327.

29. Scardamalia, M. \& Bereiter, C. (1994) "Computer Support for Knowledge-Building Communities," The Journal of the Learning Sciences, 3(3), pp. 265-283.
30. Schön, D. A. (1983) The Reflective Practitioner: How Professionals Think in Action, Basic Books, New York.

31. Simon, H. A. (1996) The Sciences of the Artificial, (Third ed.), The MIT Press, Cambridge, MA.

32. Snow, C. P. (1993) The Two Cultures, Cambridge University Press, Cambridge, UK. 